\title{
Caveolin-1 promotes radioresistance via IRGM-regulated autophagy in lung cancer
}

\author{
Xi Chen ${ }^{1,2 \#}$, Yuan-Liang Yan ${ }^{1,2 \#}$, Shuang-Shuang Zeng ${ }^{1,2}$, Zhi-Cheng Gong ${ }^{1,2}$, Zhi-Jie $\mathrm{Xu}^{3}$ \\ ${ }^{1}$ Department of Pharmacy, Xiangya Hospital, Central South University, Changsha, China; ${ }^{2}$ National Clinical Research Center for Geriatric \\ Disorders, Xiangya Hospital, Central South University, Changsha, China; ${ }^{3}$ Department of Pathology, Xiangya Hospital, Central South University, \\ Changsha, China \\ Contributions: (I) Conception and design: ZC Gong, ZJ Xu; (II) Administrative support: ZC Gong; (III) Provision of study materials or patients: ZJ \\ $\mathrm{Xu}$, X Chen, YL Yan; (IV) Collection and assembly of data: X Chen, SS Zeng; (V) Data analysis and interpretation: X Chen, YL Yan; (VI) Manuscript \\ writing: All authors; (VII) Final approval of manuscript: All authors. \\ "These authors contributed equally to this work. \\ Correspondence to: Zhijie Xu. Department of Pathology, Xiangya Hospital, Central South University, 87 Xiangya Road, Changsha 410008, China. \\ Email: xzj1322007@csu.edu.cn; Zhicheng Gong. Department of Pharmacy, Xiangya Hospital, Central South University, 87 Xiangya Road, Changsha \\ 410008, China. Email: gongzhicheng@csu.edu.cn.
}

Background: Radiotherapy is the standard therapeutic approach for non-small cell lung cancers (NSCLCs). However, radiotherapy resistance accounts for major treatment failures in NSCLC patients. Recently, targeting autophagy-related signaling has shown potential to improve radiotherapy. Furthermore, some studies have reported that caveolin-1 (Cav1), a primary scaffolding protein of caveolae, is positively associated with NSCLC progression and cell autophagy. However, the function of Cav1-mediated autophagy in NSCLC radioresistance remains largely unknown.

Methods: The NSCLC irradiation (IR)-resistant cell lines H358-IRR and A549-IRR were used for in vitro analysis. Real-time quantitative PCR (qPCR), western blot, cell counting kit-8 (CCK-8), colony formation and transmission electron microscopy analyses were performed to explore the relationship between Cav1 and immunity-related GTPase family M protein (IRGM)-regulated autophagy in the radiation resistance of lung cancers.

Results: Cav1 was significantly overexpressed in H358-IRR and A549-IRR cells compared to their parental counterparts. Knockdown of Cav1 significantly decreased the proliferation of IR-resistant NSCLC cells. Combinational treatment of IR and siRNA of Cav1 showed enhanced inhibition of the cell viability and colony formation of IR-resistant NSCLC cells. In addition, Cav1 overexpression could upregulate the autophagic proteins microtubule associated protein 1 light chain 3 II (LC3 II), Beclin-1 and Sequestosome 1 (SQSTM1/p62) in parental NSCLC cells, while Cav1 downregulation by siRNA inhibited the expression of LC3 II, Beclin-1 and p62 and the formation of autophagosomes in IR-resistant NSCLC cells. Furthermore, we observed that IRGM was downregulated after knockdown of Cav1 in IR-resistant NSCLC cells. Thus, Cav1 was observed to promote autophagy and increase IR-resistant cell survival by targeting IRGM.

Conclusions: The results of our study showed that Cav1 is involved in the development of IR resistance in NSCLC through IRGM-regulated autophagy and can be considered as a potential therapeutic target for improving the radiosensitivity of NSCLC.

Keywords: Caveolin-1; immunity-related GTPase family M protein (IRGM); autophagy; radioresistance; nonsmall cell lung cancer (NSCLC)

Submitted Apr 13, 2020. Accepted for publication 18 Sep, 2020.

doi: 10.21037/atm-20-3293

View this article at: http://dx.doi.org/10.21037/atm-20-3293

(c) Annals of Translational Medicine. All rights reserved. 


\section{Introduction}

Lung cancer is the leading cause of cancer-related deaths worldwide, with 5-year survival rates ranging from 4-17\% depending on the stage and regional differences (1). As the most common subtypes, non-small cell lung cancers (NSCLCs) can be categorized as lung adenocarcinoma (LUAD) and lung squamous cell carcinoma (LUSC), accounting for approximately $80-85 \%$ of all the cases (2). To date, the approaches used for lung cancer therapy primarily consist of surgery, chemotherapy and radiotherapy, among which radiation therapy plays a crucial role in treating all stages of NSCLC as either definitive or palliative therapy. However, the curing rates of NSCLC patients remain low, and therapeutic resistance is broadly observed (3). Thus, there is a pressing need to overcome the resistance of NSCLC, such as radioresistance. Furthermore, the use of molecular prognostication has gradually emerged into clinical therapies of NSCLC. For instance, epidermal growth factor receptor (EGFR), anaplastic lymphoma kinase, oncogenic c-ros oncogene 1 and v-raf murine sarcoma viral oncogene homolog B1 gene mutations have become predictive biomarkers for LUAD patients who could benefit from tyrosine kinase inhibitors (4). In addition, programmed death ligand 1 is a crucial marker of an effective response to immunotherapy in NSCLC patients (5-7). This evidence suggests that the identification of biomarkers associates with radiosensitivity in NSCLC patients could be a promising approach.

In NSCLC, evidence has shown that targeting autophagy-related pathways may be a promising strategy to improve the efficacy radiotherapy. The activation of the autophagy has been shown to result in an enhancement of radioresistance in a reactive oxygen species-dependent manner after treatment with low-dose irradiation (IR) (8). The ability to generate an autophagic flux and express autophagy-related proteins, including LC3 II, promotes NSCLC cell survival following IR $(9,10)$. Autophagy is a pivotal biological process that enables the disposal of dysfunctional cytoplasmic materials via sequestration of these components within autophagosomes that ultimately fuse with lysosomes for degradation (11). The function of autophagy is essential for intracellular homeostasis maintenance and self-protection in all human cells (12-14). The process of autophagy is controlled by a series of signaling events that involve numerous evolutionarily conserved genes called autophagy-related genes (ATGs). The initiation of autophagy is regulated by the serine/ threonine-protein kinase unc-51-like kinase 1 (ULK1) complex. Subsequently, a class III PI3K complex, scaffolded by Beclin-1, is activated for nucleation of the autophagosomes. In addition, two ubiquitin-like conjugation systems, the ATG12-ATG5 and Microtubule associated protein 1 light chain 3 (LC3) systems, mediate the eventual formation of autophagosomes. The formation ATG12-ATG5 complex is catalyzed by the E1-like enzyme ATG7 and the E2-like enzyme ATG10 and is induced to expand autophagosome membranes. Furthermore, LC3 is cleaved by ATG4 and subsequently binds to the soluble protein LC3 I, which conjugates phosphatidylethanolamine generate LC3 II. LC3 II binds autophagosome membranes and serves as a marker of mature autophagosomes. During fusion with the lysosome, LC3 II is degraded along with other cargo of autophagosomes, resulting in turnover of cell components and promoting cellular self-protection (15-17). Sequestosome 1 (SQSTM1/p62) could severe as a signaling hub which interacts with LC3, and is degraded by autophagy (18). In addition, p62 overexpression has been proposed to increase selective autophagy through modulating ubiquitin signaling (19),

Caveolin-1 (Cav1) is a scaffolding protein of caveolae on the plasma membrane that has been shown to be closely associated with oncogenesis and malignancy development. Elevated expression of Cav1 has been confirmed to promote the proliferation, differentiation and migration of cells of various cancers, such as lung, pancreatic, and breast cancers (20-22). With respect to radioresistance, IR upregulates the expressions of Cav1 in pancreatic, breast and lung cancers (23-27). In NSCLC, following IR, phosphorylated Cav1 at Tyr14 has been shown to promote EGFR nuclear translocation, a process through which enhances residual DNA damage is blocked (26). Among radiotherapy-treated patients with brain metastasis from NSCLC, an increased risk of death was observed in a group with higher Cav1 expression (28). Currently, although some findings have suggested a potential role of Cav1 in amplified resistance to IR, the mechanisms by which Cav1 is involved in the IR resistance of NSCLC remains to be elucidated. A previous study shows that Cav1 deficiency induces autophagy and lysosomal function in breast cancers via disruption of cell membrane-located lipid rafts, which is considered to be a cell survival mechanism for overcoming nutritional stress (29). In contrast, another study reports that oncogenic Cav1 contributes to hepatocellular carcinoma cell survival by inhibiting autophagy (30). In lung cancers, knockdown of Cav1 can augment the therapeutic sensitivity to cisplatin by 
inhibiting mitophagy (31). At present, there are conflicting reports regarding the role of Cav1 in the regulation of autophagy in cancer cells, and the mechanisms through which Cav1 involvement in the regulation of radioresistance is correlated with autophagy remains unelucidated.

Therefore, in this study, we set out to elucidate the autophagy-related mechanisms of Cav1 associated with radiation resistance in NSCLC. We provide evidence for a previously unknown mechanism in which upregulation of Cav1 promotes IR-resistant NSCLC cell proliferation via immunity-related GTPase family $M$ protein (IRGM)-regulated autophagy. Our data provide a better understanding of the role of Cav1 in the regulation of radiation resistance and indicates that Cav1 could be used as a novel therapeutic target to improve the sensitivity of radiation therapy in human NSCLC.

\section{Methods}

\section{Cell culture}

The NSCLC parental cell lines H358-P (ATCC Number: CRL-5807) and A549-P and the IR-resistant cell lines H358-IRR and A549-IRR were kind gifts from the lab of Prof. Deng (32). The cell lines H358-P/H358-IRR and A549-P/A549-IRR were cultured under aseptic conditions using 1640 medium (11875500BT, Gibco, USA), supplemented with $10 \%(\mathrm{v} / \mathrm{v})$ fetal bovine serum (04-001$1 \mathrm{~A} / \mathrm{B}$, BioInd, Israel) and $1 \%$ penicillin and streptomycin. All cell lines were cultured in a humidified incubator at $37^{\circ} \mathrm{C}$ under an atmosphere with $5 \% \mathrm{CO}_{2}$.

\section{Plasmid and small interfering $R N A$ transfection}

A Cav1 overexpression plasmid was constructed by cloning human Cav1 cDNA into the plasmid pcDNA3.1 (pc3.1Cav1). An IRGM overexpression plasmid (IRGM-pc3.1) was purchased from MiaoLingPlasmid (Wuhan, China). The empty plasmid pcDNA3.1 served as the vector control. Small interfering RNAs (siRNAs) targeting Cav1 (si-Cav1, 5'-AGACGAGCUGAGCGAGAAGCA-3') were custom synthesized by RiboBio (Guangzhou, China). A scrambled siRNA purchased from RiboBio was used as a negative control. Cells were seeded in six-well plates at a density of $1 \times 10^{5}$, and transfection was performed using Lipofectamine 3000 reagent (L3000015, Invitrogen, USA) according to the manufacturer's protocol.

\section{RNA Extraction and Quantitative PCR (qPCR)}

Total RNA was extracted from NSCLC cell lines using TRIzol reagent (Invitrogen, USA) according to the manufacturer's protocol and then converted to cDNA using a PrimeScriptTM RT reagent kit (6210, Takara, China). The qPCR assay was performed with iTaqTM Universal SYBR green Supermix (1725121, Bio-Rad, USA), and $\beta$-actin was used as an internal control. The sequences of the forward and reverse primers used in this study are provided in Table S1. As the previous reports (33) indicated that the relative expression levels were determined using the $2^{-\triangle \Delta C T}$ method, and all reactions were performed three or more times.

\section{Western blot analysis}

Whole cell lysates were prepared with RIPA buffer supplemented with protease and phosphatase inhibitor cocktails (B14012, Bimake, USA). Protein concentrations were determined using the BCA protein assay (23229, Thermo Fisher Scientific, USA). Equal amounts of proteins were subjected to SDS-polyacrylamide gel electrophoresis and then transferred to the appropriate PVDF membranes (0.22 $\mu \mathrm{m}$ : ISEQ00010; $0.45 \mu \mathrm{m}$ : IPVH00010, Millipore, USA). The membranes were blocked with $5 \%$ nonfat dry milk at room temperature for $1 \mathrm{~h}$ and then incubated overnight at $4{ }^{\circ} \mathrm{C}$ with primary antibodies against Caveolin1(16447-1-AP, Proteintech, USA), LC3 A/B (4108S, Cell Signaling Technology, USA), Beclin-1 (3495S, Cell Signaling Technology, USA), IRGM (AP11128b, Abgent, USA), p62 (sc-28359, Santa, USA) and $\beta$-actin (sc-58673, Santa, USA). Protein bands were visualized through Immobilon Western chemiluminescent reagents (WBKLS0500, Millipore, USA).

\section{Transmission electron microscopy (TEM)}

Cells were plated in six-well plates and transfected with siRNA or plasmids for $48 \mathrm{~h}$. Subsequently, the cells were digested with trypsin, collected into a pellet, and then double-fixed in a $2.5 \%$ glutaraldehyde solution with Millonig's phosphate buffer ( $\mathrm{pH}=7.3$ ) before being shipped overnight at ambient temperature to the TEM laboratory at the Pathology Department of Xiangya Hospital, Changsha, Hunan, where cells were processed as follows. The cells were washed three times at $10 \mathrm{~min}$ intervals 
with Millonig's phosphate buffer, incubated for $1 \mathrm{~h}$ in $1 \%$ osmium tetroxide and then washed three times at $10 \mathrm{~min}$ intervals with Millonig's phosphate buffer. Dehydration of the cells was performed at room temperature in a graded series of $50 \%, 70 \%$, and $90 \%$ acetone at $10 \mathrm{~min}$ intervals for each step followed by two incubations in $100 \%$ acetone at $15 \mathrm{~min}$ intervals. The resin soaking and embedding process was performed with the specimens using an 1:1 mix of acetone:resin for $12 \mathrm{~h}$, and $100 \%$ resin was used to polymerize overnight at $37^{\circ} \mathrm{C}$. The resin solidifying process involved treating the cells with $100 \%$ resin to polymerize overnight at $37^{\circ} \mathrm{C}$ followed by incubation for $12 \mathrm{~h}$ at $60^{\circ} \mathrm{C}$. Subsequently, 50-100 nm ultrathin sections of cells were made with an ultramicrotome and a diamond knife. After 3\% uranyl acetate and lead nitrate double staining, the cells were examined and imaged on a electron microscope (HT-7700, Hitachi, Japan).

\section{CCK-8 assays}

Cell growth and viability were analyzed using a CCK-8 assay. In brief, cells were seeded in 96-well plates at a density of 800 cells per well after transfection for $12 \mathrm{~h}$. Then, the cells were incubated at $37{ }^{\circ} \mathrm{C}$ under an atmosphere with $5 \%$ $\mathrm{CO}_{2}$ for $1 \mathrm{~h}$ after adding the CCK-8 test solution (B34304, Bimake, USA). Subsequently, the optical density (OD) of the samples was measured at $450 \mathrm{~nm}$ using a spectrometer (PerkinElmer, United States).

\section{Colony formation assay}

The protocols used for the colony formation assay have been described previously (34). In brief, cells were seeded in 6-well plates at a density of 1,000 cells per well, and after incubating for $24 \mathrm{~h}$, the cells were treated with different doses of IR using a gamma irradiator. After approximately 2 weeks, colonies were stained with $0.3 \% \mathrm{w} / \mathrm{v}$ crystal violet/ methanol for $20 \mathrm{~min}$ at room temperature.

\section{Computational survival analysis}

The correlation between Cav1 gene expression and prognosis was analyzed in the publicly available UALCAN platform (http://ualcan.path.uab.edu), which was based on the TCGA (The Cancer Genome Atlas) data of LUAD and LUSC patients.

\section{Statistical analysis}

All experiments were performed at least in triplicate and are reported as the means $\pm \mathrm{SD}$, the differences between which were analyzed for significance using Student's $t$-test for pairwise comparisons or ANOVA for multivariate analysis. Data analysis was performed using GraphPad Prism 5 and SPSS 23.0. Differences were considered significant at *, $\mathrm{P}<0.05 ;{ }^{* *}, \mathrm{P}<0.01$; ***, $\mathrm{P}<0.001$. for all tests.

\section{Results}

\section{Identification of radioresistant phenotypes of NSCLC cells}

To determine whether the radioresistant phenotypes of NSCLC cells had been maintained, H358-P/H358-IRR and A549-P/A549-IRR cells were exposed to different doses of IR (0, 2, 4 and 6 Gy). Subsequently, all cell lines were subjected to colony formation assays to assess cellular proliferation rates. The results showed that the colony formation rates of the H358-IRR and A549-IRR cells after IR were significantly higher than those observed for the H358-P and A549-P cells (Figure 1). These results are consistent with the radioresistant phenotype of the H358IRR and A549-IRR cell lines.

\section{Prognostic analysis based on Cav1 expression in LUAD and LUSC patients}

To assess the relationship between Cav1 expression and prognosis, a LUAD dataset (502 cases, including 122 high expression and 380 medium/low expression cases) and a LUSC dataset (494 cases, including 125 high expression and 369 medium/low expression cases) from TCGA were used to perform survival analysis. The survival curve results revealed that patients with high tumoral Cav1 expression had remarkably decreased survival probability in the LUAD and LUSC patients (Figure 2; $\mathrm{P}=0.027$ and 0.017, respectively).

\section{Cav1 induces autophagy in IR-resistant NSCLC cells}

Based on two verified cell lines, we examined the expression levels of Cav1 in parental and IR-resistant cells. Through qPCR and western blot assays, elevated mRNA and protein levels of Cav1 were detected in H358-IRR and A549IRR cells compared to their parental cells $(\mathrm{P}<0.01$ for all 
A

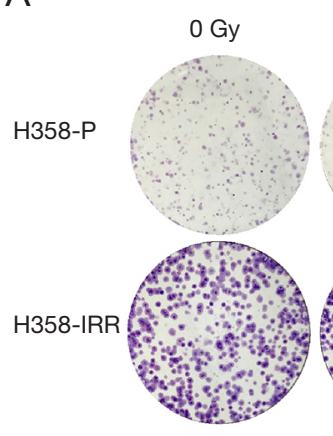

2 Gy

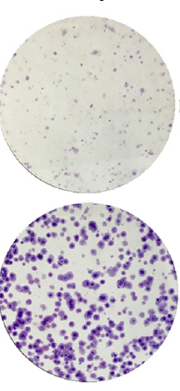

4 Gy

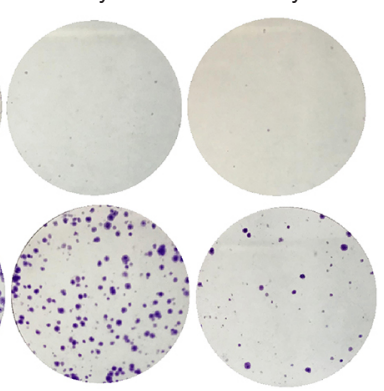

C

0 Gy

2 Gy

4 Gy

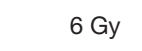

A549-P
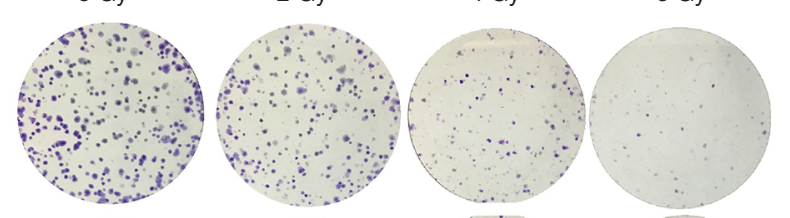

A549-IRR
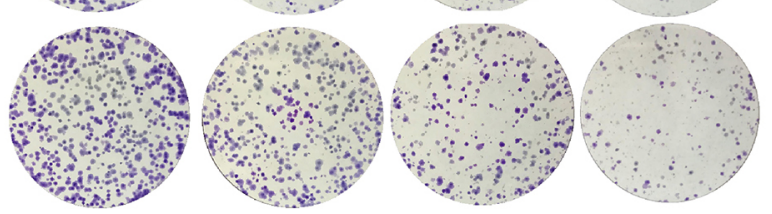

B
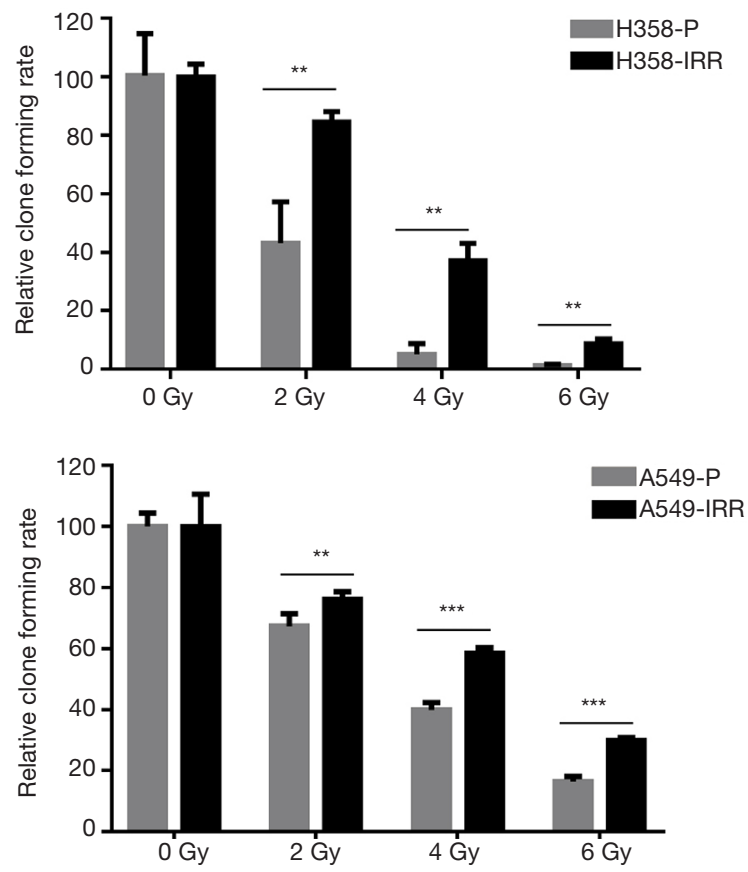

Figure 1 Confirmation of the IR-resistant phenotypes of NSCLC H358-IRR and A549-IRR cells. (A,B) The colony formation assays for the H358-P and H358-IRR cell lines treated with different doses of IR. (C,D) The colony formation assays for A549-P and A549IRR cell lines treated with different doses of IR. The colonies were dyed with crystal violet and photographed under the camera $(\times 1)$. The quantitative results are performed in three independent experiments. ${ }^{* *}, \mathrm{P}<0.01$; ${ }^{* * *}, \mathrm{P}<0.001$.

A

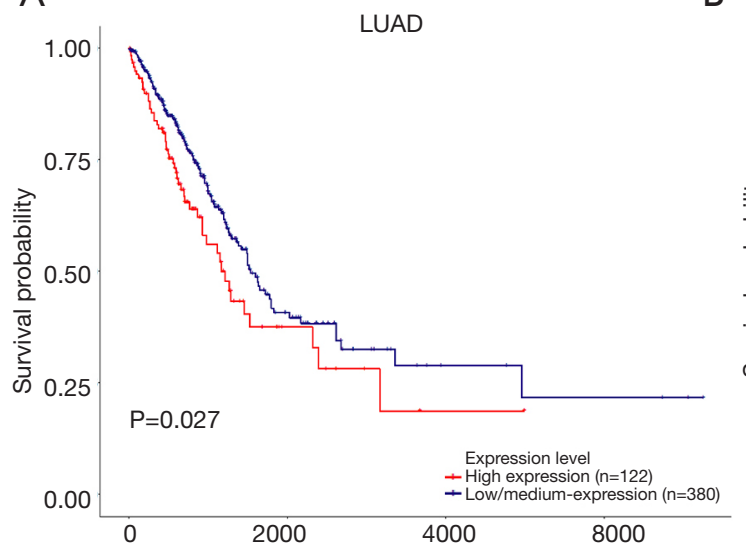

B

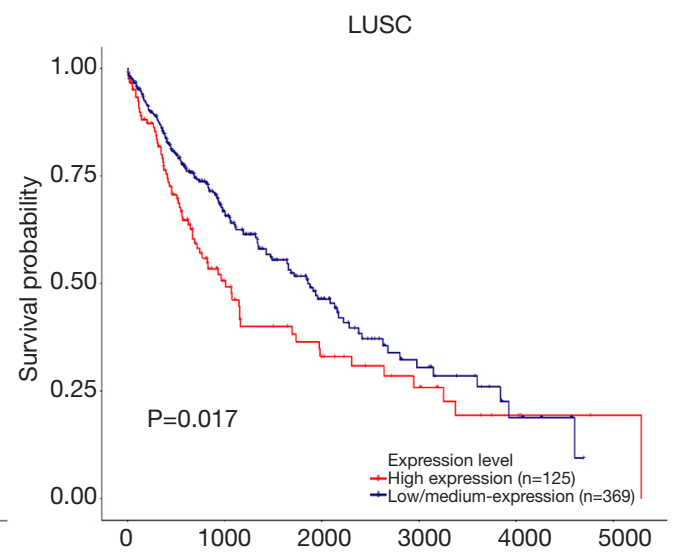

Figure 2 Prognostic role of Cav1 in LUAD and LUSC patients. (A) The survival curve was plotted to compare the survival probability between high and low/medium expression of Cav1 in LUAD patients. (B) The survival curve was plotted to compare the survival probability between the high and low/medium expression of Cav1 in LUSC patients. LUAD, lung adenocarcinoma; LUSC, lung squamous cell carcinoma. 
comparisons; Figure $3 A, B)$. Next, to investigate the levels of autophagy, we compared key markers of autophagy (LC3 II, Beclin-1 and p62) in parental and IR-resistant cells (Figure $3 A$ ). The results showed that compared to the H358-P and A549-P cells, the protein expression levels of LC3 II, Beclin-1 and p62 were increased in the H358IRR and A549-IRR cells. Furthermore, the expression of LC3 II, Beclin-1 and p62 were verified in LUAD tissues compared with those of the normal tissues from Human Protein Atlas project (http://www.proteinatlas.org) (35). The increased levels of LC3 II (encoded by MAP1LC3B), Beclin-1(encoded by BECN1) and p62 (encoded by the gene SQSTM1) are moderately expressed in LUAD samples but weakly expressed in normal lung specimens (Figure 3C). To probe the relationship between expression of above autophagic markers and prognosis of LUAD patients, Kaplan-Meier plotter platform (http://kmplot. com/analysis/) (36) were used to conduct survival analysis. The results showed that patients with high levels of LC3 II, Beclin-1 and p62 had significantly shorter overall survivals (Figure S1).

To further investigate whether Cav1 is involved in regulating autophagy, a dataset of 526 LUAD patients (Dataset ID: TCGA-LUAD.htseq_counts.tsv, with a total of 585 analyzed samples and 59 adjacent nontumor tissues were excluded) from UCSC xena platform (https://xenabrowser.net/datapages/) were download to analyze the correlation between expression of Cav1 and above autophagic markers. The results showed that expression levels of Cav1 was positively associated with expression of LC3 II, Beclin-1 and p62 (Figure 3D). Then we overexpressed Cav1 in H358-P and A549-P cells and knocked down Cav1 expression in H358-IRR and A549IRR cells. We observed that transfection of H358-P and A549-P cells with the Cav1-pc3.1 plasmid upregulated the expression of LC3 II, Beclin-1 and p62 (Figure 3E). Furthermore, silencing Cav1 by siRNA in IR-resistant cells reduced the protein expression of LC3 II, Beclin-1 and p62 (Figure $3 F$ ). Consistently, TEM analysis results showed that downregulation of Cav1 inhibited the formation of autophagosomes $(\mathrm{P}<0.05$ for all comparisons; Figure $3 G)$. Taken together, the above findings suggest that Cav1 can promote autophagy in NSCLC cells.

\section{Knockdown of Cav1 increases the sensitivity of IR-resistant NSCLC cells}

Next, to explore the effects of Cav1 in IR-resistant
NSCLC cells, we knocked down Cav1 expression in H358IRR and A549-IRR cells and examined cell proliferation through CCK-8 assays. Compared to cells transfected with scrambled siRNA, cells transfected with siCav1 exhibited decreased proliferation (Figure 4A). Furthermore, to assess functions of Cav1 on NSCLC radioresistance, H358-IRR and A549-IRR cells were treated with 0 or 4 Gy IR after knockdown of Cav1, and CCK-8 assays were performed to examine cell viability. The results showed that cells having undergone the combined treatment of siCav1 and 4 Gy IR exhibited notably decreased viability compared to those treated with 4 Gy alone $(\mathrm{P}<0.01$; Figure $4 B)$. Furthermore, we investigated the role of Cav1 in the resistance of NSCLC to IR through colony formation assays. The results showed that the rate of relative colony formation in the group with the combined treatment of siCav1 and 4 Gy was significantly lower than observed in the group treated with IR alone (Figure 4C). Taken together, these data indicate that downregulating Cav1 expression could be a valid approach to enhance the sensitivity of IR-resistant NSCLC cells.

\section{Cav1 enhances autophagy by upregulating IRGM in IR- resistant NSCLC cells}

To further investigate the potential downstream molecules involved in Cav1-regulated autophagy, we compared the mRNA expression profiles between the siCav1 and paired control groups in H358-IRR and A549-IRR cells using an autophagy $\mathrm{RT}^{2}$ Profiler ${ }^{\mathrm{TM}}$ PCR array. The detailed primer sequences of the array are listed in Table S1. The results showed that among the expression profiles of 84 autophagyrelated genes (Table S2), 17 genes showed significant differential expression in H358-IRR or A549-IRR cells (Figure $5 A, B)$. However, only IRGM showed downregulated mRNA levels in H358-IRR and A549-IRR cells after knockdown of Cav1, with more than a 2 -fold change observed (Figure $5 A, B$ ). Subsequently, we validated the protein level of IRGM by western analysis, and the results revealed that the IRGM protein levels were consistent with those observed for mRNA levels in the $\mathrm{qPCR}$ array $(\mathrm{P}<0.01$ for all comparisons; Figure 5C,D).

Furthermore, after Cav1 knockdown, decreased IRGM expression was accompanied by downregulation of the autophagic proteins LC3 II, Beclin-1 and p62 (Figure 5D). Since IRGM can regulate the initiation of autophagy (37), we postulated that the Cav1-RGM axis mediates autophagy in IR-resistant NSCLC cells. 
A

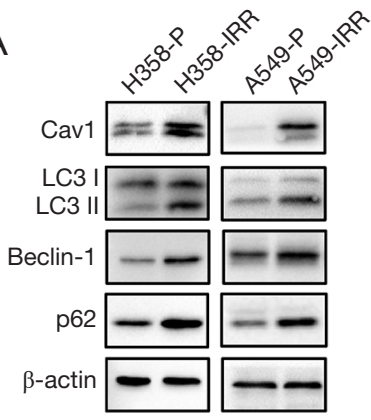

B

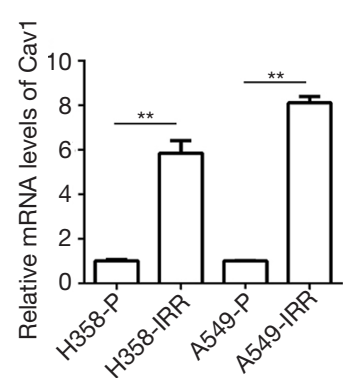

C

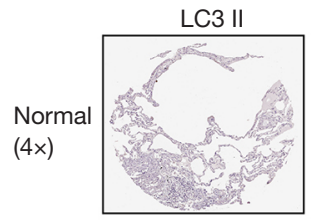

LUAD

$(4 \times)$

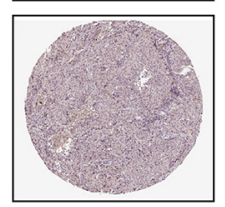

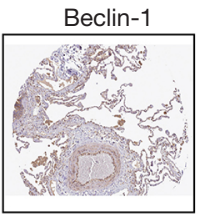

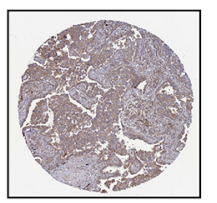

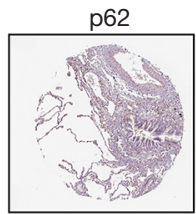

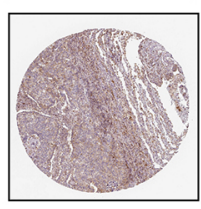

$\mathrm{E}$
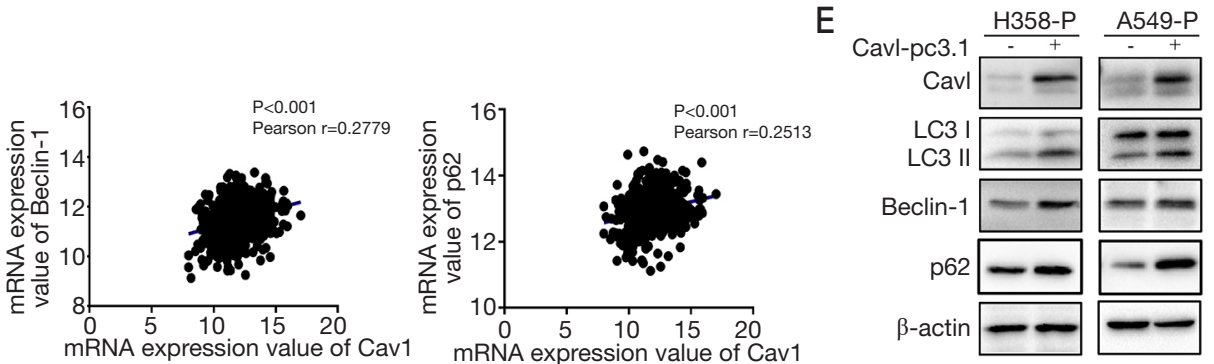

$\mathrm{F}$

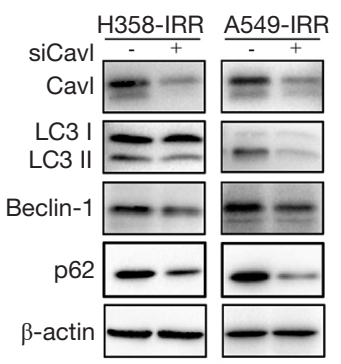

G

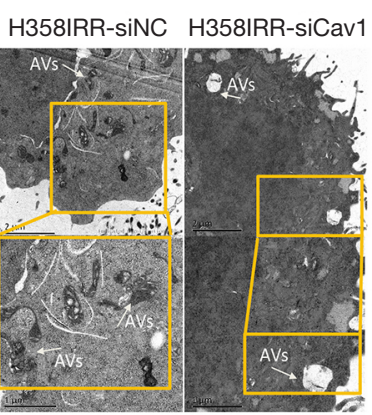

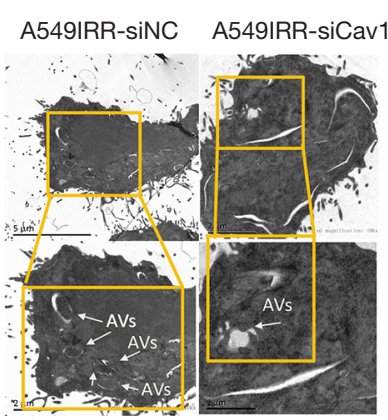

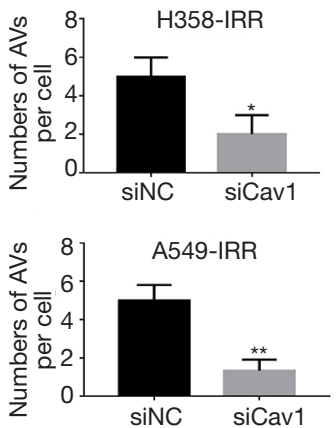

Figure 3 Cav1 promotes autophagy in parental and IR-resistant NSCLC cells. (A) Protein levels of Cav1, LC3 II, Beclin-1 and p62 were detected by western blot analysis in H358-P/H358-IRR, A549-P/A549-IRR cells. (B) Relative mRNA levels of Cav1 were examined with a qPCR array in H358-P/H358-IRR and A549-P/A549-IRR cells. (C) The Human Protein Atlas project shows the representative immunohistochemical images of LC3 II, Beclin-1 and p62 in LUAD compared with normal lung tissues. The staining method was described according to annotations in Human Protein Atlas project (https://www.proteinatlas.org/about/assays+annotation\#ih_annotation). (D) The correlation between Cav1 expression levels and LC3 II, Beclin-1 and p62 levels. (E) H358-P and A549-P cells were transfected with pcDNA3.1 or pcDNA-Cav1, after which LC3 II, Beclin-1 and p62 protein levels were assessed by western blot analysis. (F) H358-IRR and A549-IRR cells were transfected with siNC or siCav1, followed by the detection of Cav1, LC3 II, Beclin-1 and p62 protein levels. (G) After siNC or siCav1 transfection of H358-IRR and A549-IRR cells, autophagosome formation was detected by TEM analysis. *, P<0.05; **, $\mathrm{P}<0.01$. LUAD, Lung adenocarcinoma, Cav1, Caveolin-1; LC3 II, Microtubule associated protein 1 light chain 3 II; p62, Sequestosome 1; Avs, autophagic vacuoles. 
A

H358-IRR

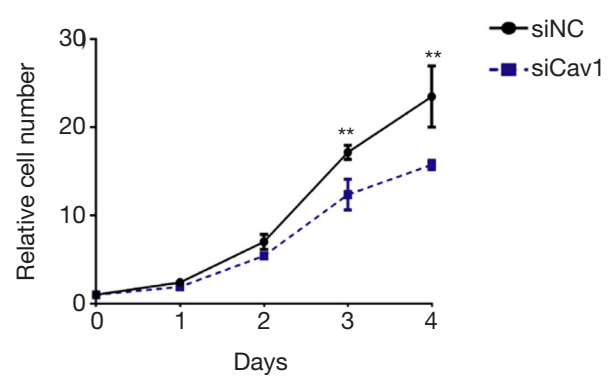

B H358-IRR

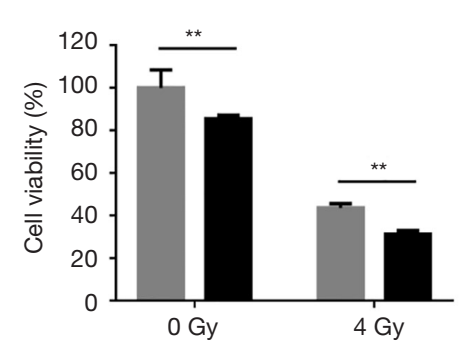

C

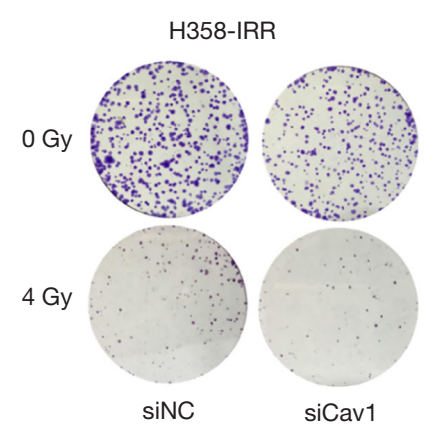

A549-IRR

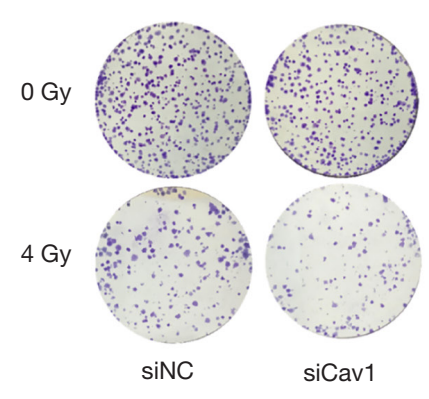

A549-IRR

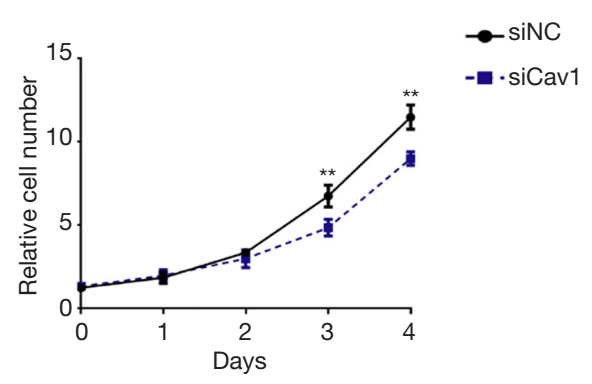

A549-IRR

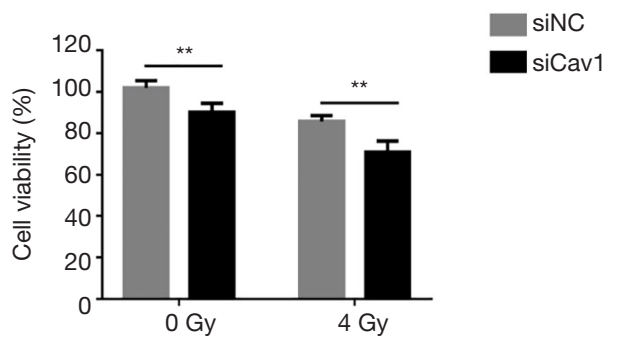

H358-IRR

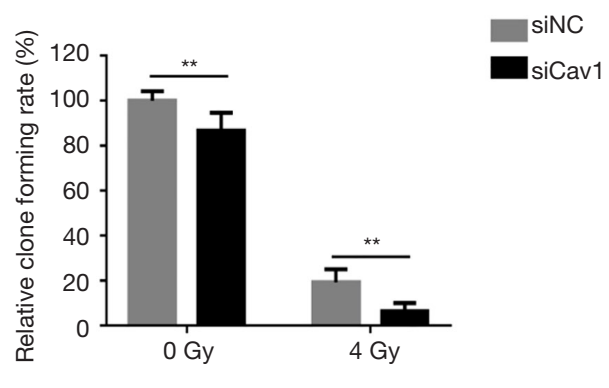

A549-IRR

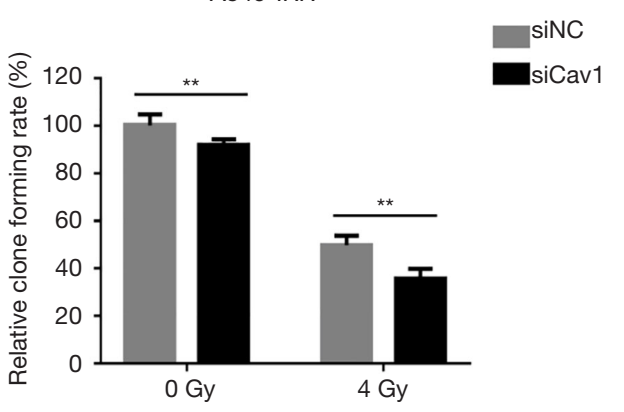

Figure 4 Cav1 modulates the IR sensitivity of IR-resistant NSCLC cells. (A) After transfection of siNC or siCav1 in H358-IRR and A549IRR cells, CCK-8 assays were performed to examine the cell growth for 4 days. (B) H358-IRR and A549-IRR cells transfected with siCav1 or siNC were exposed to 4 Gy, and after 96 h were assessed by CCK-8 assays to detect cell viability. (C) H358-IRR and A549-IRR cells transfected with siCav1 or siNC were exposed to $4 \mathrm{~Gy}$ and evaluated in colony formation assays. The colonies were dyed with crystal violet and photographed under the camera $(\times 1)$. ${ }^{* *}, \mathrm{P}<0.01$. The experiments were performed three times with similar results. 
A

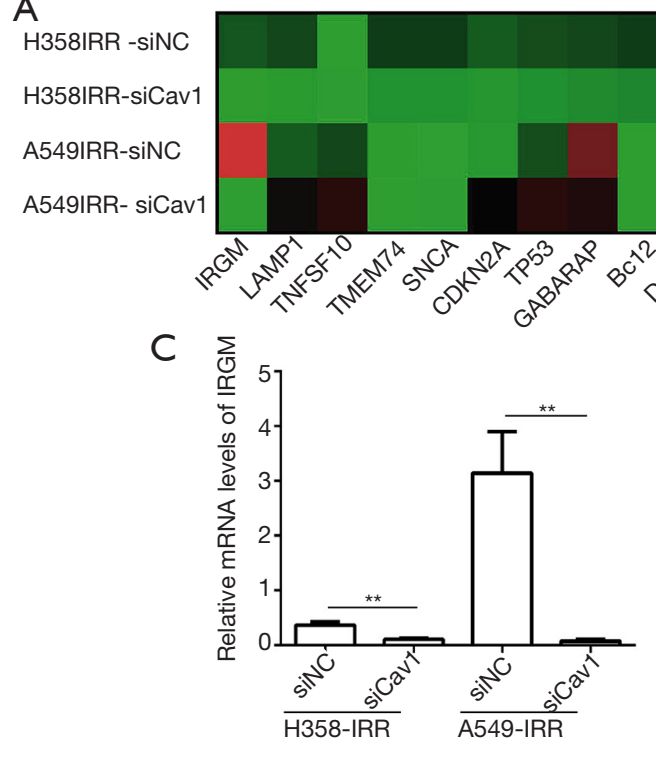

$\mathrm{F}$

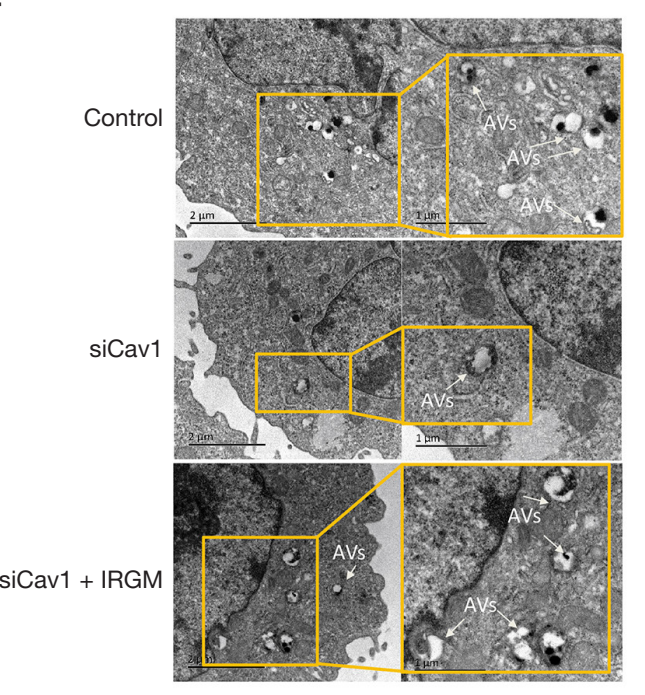

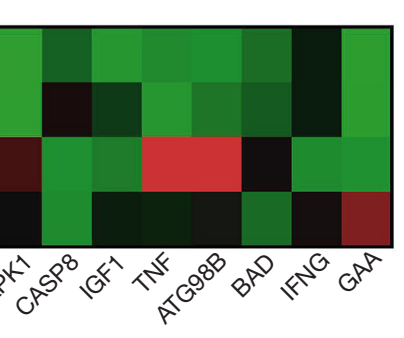

D

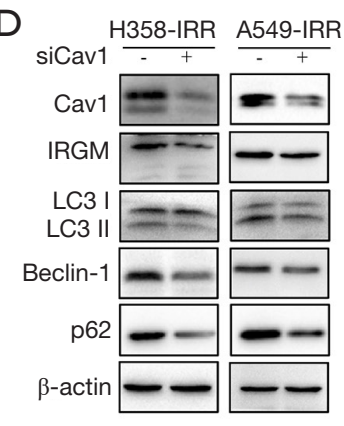

A549-IRR

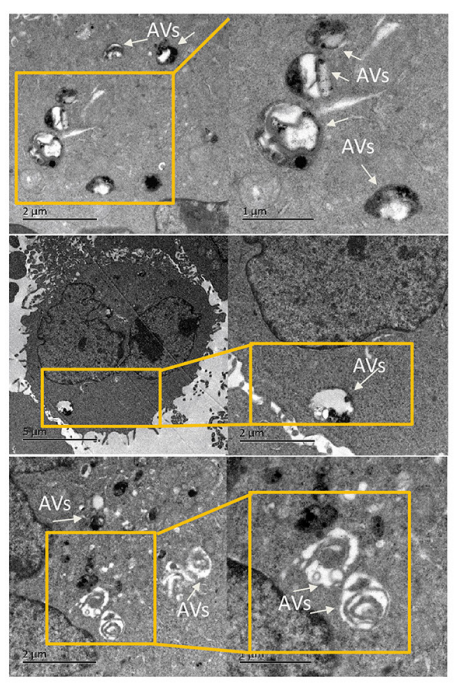

E
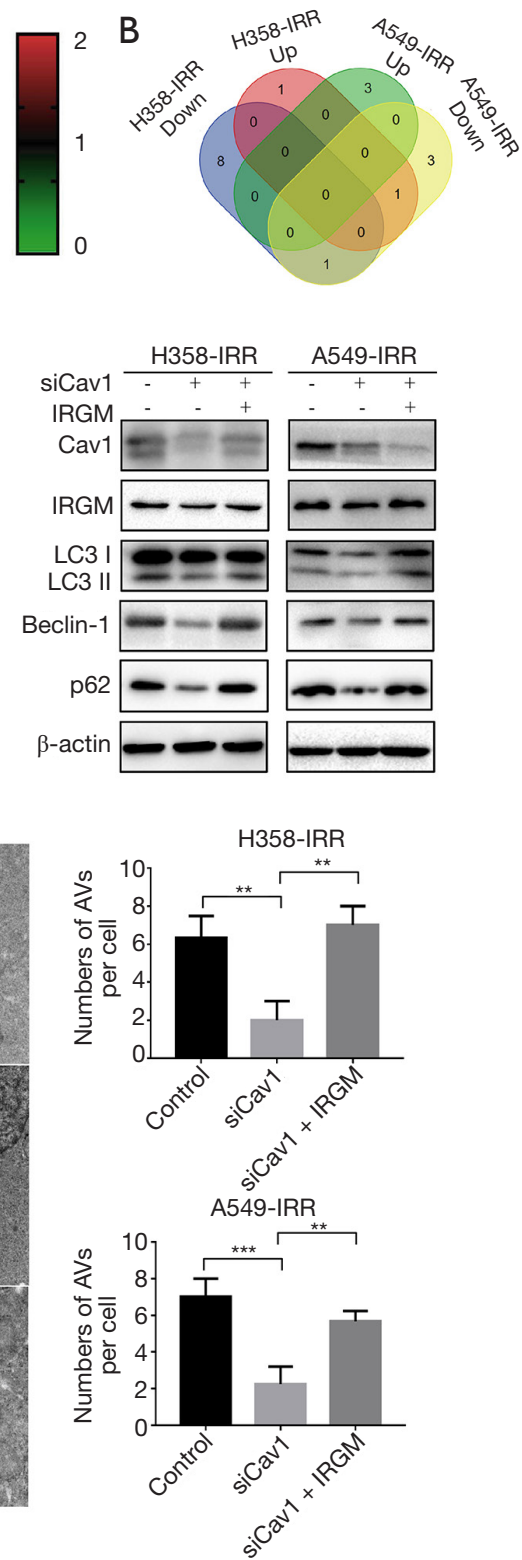

Figure 5 Cav1-IRGM axis promotes autophagy in IR-resistant NSCLC cells. (A) H358-IRR and A549-IRR cells transfected with siCav1 or siNC were assessed using an autophagy RT2 Profiler ${ }^{\mathrm{TM}}$ PCR array. The heatmap shows that 17 genes were identified with $>2$-fold mRNA differential expression in H358-IRR and A549-IRR cells. (B) A Venn diagram was used to show that IRGM was the only gene with >2-fold mRNA differential expression in both IR-resistant cell lines. (C) IRGM mRNA expression was assessed in H358-IRR and A549-IRR cells transfected with siCav1 or siNC. (D) After transfection with siCav1 or siNC, the protein expression of Cav1, IRGM, LC3 II, Beclin-1 and p62 was detected in H358-IRR and A549-IRR cells by western blot analysis. (E) The reverse experiment was performed by the successive knockdown of Cav1 and overexpression of IRGM in H358-IRR and A549-IRR cells. Through western blot assays, we examined the expression of IRGM, Cav1, LC3 II, Beclin-1 and p62. (F) Based on the experimental regimens described above, autophagosomes were analyzed by TEM analysis. ${ }^{* *}, \mathrm{P}<0.01$; ${ }^{* * *}, \mathrm{P}<0.001$. Experiments were performed three times with similar results. Cav1, Caveolin-1; LC3 II, Microtubule associated protein 1 light chain 3 II; p62, Sequestosome 1; IRGM, Immunity-related GTPase family M protein LAMP1,Lysosomal associated membrane protein 1; TNFSF10, Tumor necrosis factor (ligand) superfamily member 10; TMEM74, Transmembrane protein 74; SNCA, $\alpha$-Synuclein; CDKN2A, Cyclin-dependent kinase inhibitor 2A; GABARAP, GABA-type A receptor-associated protein; Bcl2, B-cell lymphoma 2; DAPK1, Death-associated protein kinase 1; CASP8, Caspase-8; IGF1, Insulin-like growth factor-1; TNF, Tumor necrosis factor; ATG9B, Autophagy-related protein 9B; BAD, Bcl-2-associated death promoter; IFNG, Interferon gamma; TP53 Tumor protein 53; GAA, acid- $\alpha$-glucosidase. Avs: autophagic vacuoles. 
A

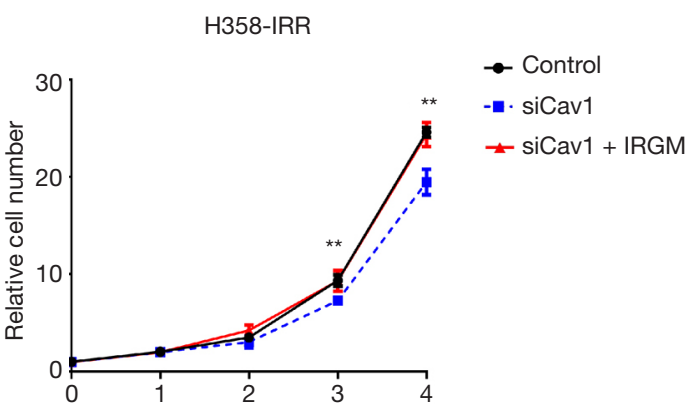

B

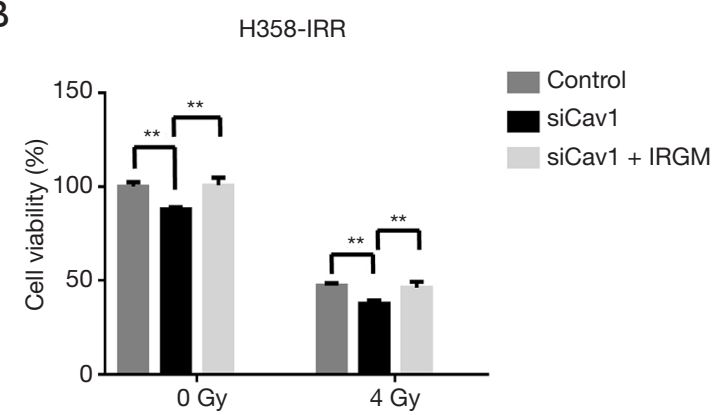

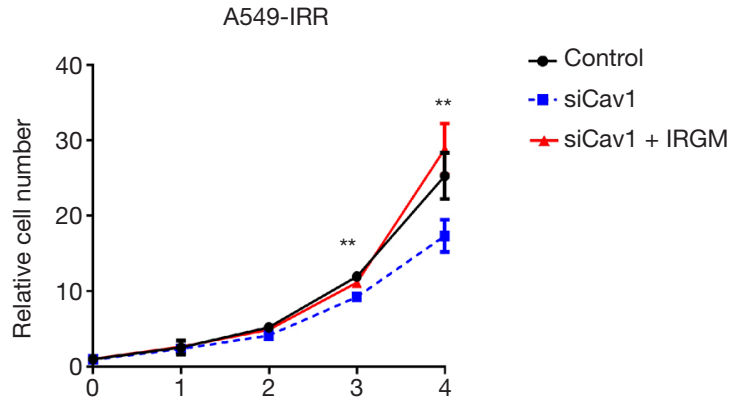

A549-IRR

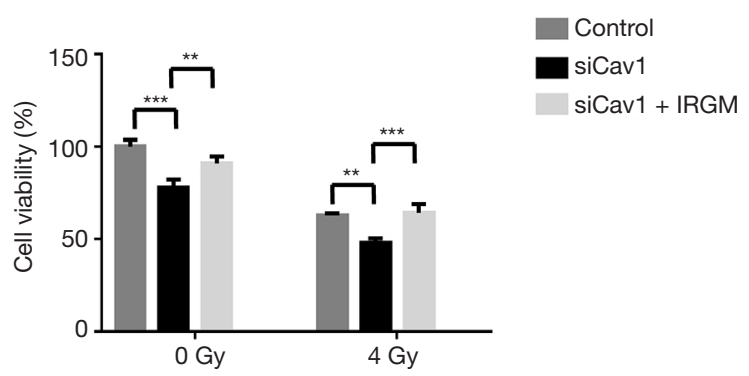

Figure 6 Knockdown of the Cav1-IRGM axis improves the survival of IR-resistant NSCLC cells. (A) Through CCK-8 assays, cell growth curves were plotted in H358-IRR and A549-IRR cells. (B) After treatment of siNC or siCav1 with pc3.1-IRGM or pcDNA3.1, the cells were exposed to 0 or 4 Gy, and cell viability was measured after 96 h by CCK- 8 assays. ${ }^{* *}, \mathrm{P}<0.01$; ${ }^{* * *}, \mathrm{P}<0.001$. Experiments were performed three times with similar results.

Therefore, we conducted an experiment in which we transfected H358-IRR and A549-IRR cells with siCav1 or scrambled siRNA, and after $24 \mathrm{~h}$, we transfected the siCav1-pretreated cells with pc3.1-IRGM or pcDNA3.1. Subsequently, western blot results showed that without upregulation of IRGM, downregulation of Cav1 suppressed LC3 II, Beclin-1 and p62 expression, whereas overexpression of IRGM reversed the downregulated expression of LC3 II, Beclin-1 and p62 caused by Cav1 knockdown. (Figure 5E). Consistent with the western blot data, TEM analysis results showed that the formation of autophagosomes was significantly blocked by siCav1, and the inhibited generation of autophagosomes was reverted to levels observed in blank controls after upregulation of IRGM. (Figure 5F; $\mathrm{P}<0.01$ for all comparisons). Taken together, these data support the hypothesis that Cav1 induces IRGM-related autophagy in IR-resistant NSCLC cells.

\section{Knockdown of the Cav1-IRGM axis sensitizes IR-resistant NSCLC cells}

To investigate whether the inhibition of cell proliferation was caused by the Cav1-IRGM axis, we performed an experiment using H358-IRR and A549-IRR cells in which down regulation of Cav1 was observed to decrease the proliferation of H358-IRR and A549-IRR cells, and this suppression was reversed by upregulating IRGM $(\mathrm{P}<0.01$; Figure $6 \mathrm{~A}$ ). Furthermore, we evaluated the role of the Cav1-IRGM axis in NSCLC cell viability after IR. The results showed that IRGM overexpression significantly rescued cell viability that was impaired by Cav1 knockdown after exposure to IR at $4 \mathrm{~Gy}$. ( $\mathrm{P}<0.01$ for all comparisons; Figure $6 B$ ). Taken together, these findings suggest that Cav1 knockdown promotes cell killing by targeting IRGM.

\section{Discussion}

In the present study, we demonstrated the role of Cav1 in IR-resistant NSCLC cells. Higher Cav1 levels were observed in cells with stronger resistance to IR, while knockdown of Cav1 significantly enhanced the radiosensitivity of IR-resistant NSCLC cells. These findings are accordance with evidence showing that in NSCLC, Cav1 promoted IR-induced cell survival and increased the 


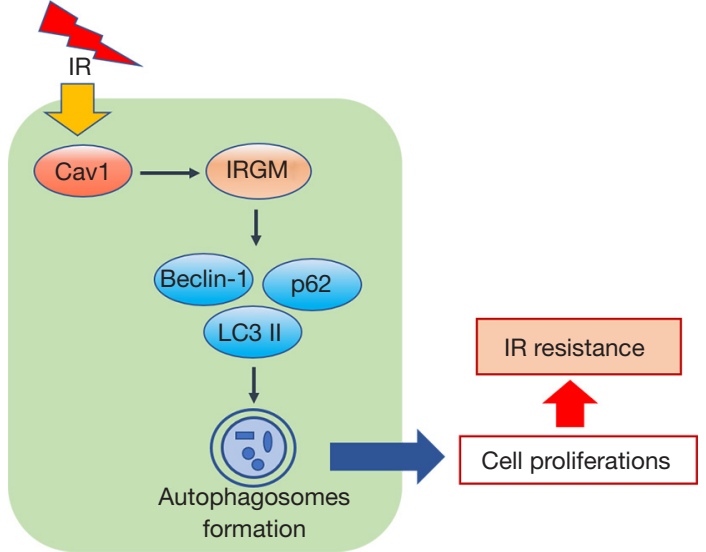

Figure 7 Schematic illustrating that IR resistance is induced by Cav1 through the activation of IRGM-mediated autophagy. Upregulated Cav1 has pro-growth effects by activating IRGMmediated autophagy, resulting in the IR resistance phenotype in NSCLC cells. Cav1, Caveolin-1; LC3 II, Microtubule associated protein 1 light chain 3 II; p62, Sequestosome 1; IRGM, Immunityrelated GTPase family $\mathrm{M}$ protein.

risk of death in IR-treated patients $(26,28)$. Thus, our data reaffirm that targeting the activity of Cav1 may be a novel therapeutic approach for the treatment of radioresistant NSCLCs.

Autophagy is well known to be an important biological function process involving in several human disorders due to its acquired dual roles in cell survival and death processes (38-40). Due to conflicting data, the functions of autophagy in IR resistance remain poorly understood. In the present study, for the first time, we uncovered the Cav1mediated cytoprotective role of autophagy in IR-resistant NSCLC (Figure 7). We observed that in two IR-resistant NSCLC cell lines, LC3 II, Beclin-1 and p62 levels were significantly higher than those observed in paired parental cells. Furthermore, the overexpression of Cav1 in parental cells promoted the expression of LC3 II, Beclin-1 and p62, while knockdown of Cav1 decreased LC3 II, Beclin-1 and p62 levels and the number of autophagosomes in IRresistant NSCLC cells. Since LC3 II, Beclin-1 and p62 are essential mediators of autophagosome formation (41), our data provide a possible explanation of how Cav1 overcomes IR-induced impairment through pro-survival functions of autophagy.

Furthermore, for the first time, we elucidated the role of the Cav1-IRGM axis in IR-resistant NSCLC cells. IRGM is a member of the interferon-inducible GTPases
(IRGs), a powerful cell-autonomous system used to defend against intracellular pathogens (42). To date, IRGM is primarily known for its positive regulation of autophagy in some inflammatory and infectious disorders. Several studies have demonstrated that IRGM induces the initiation of autophagy by interacting with phosphorylated ULK1 and Beclin-1 (37). In addition, IRGM can inhibit inflammasome activation by promoting the p62-mediated selective autophagic degradation of the inflammasome component NLRP3 (43). Although the links between IRGM and autophagic machinery are well-established in inflammatory and infectious diseases, the contributions of IRGM to autophagy in tumor progression have remained unclear. To date, IRGM has only been observed to promote tumor development in hepatocellular carcinoma, gastric cancer (44), melanomas and gliomas (45). Knockdown of IRGM can suppress the expressions of LC3 II and p62 in gliomas (45) and melanomas (46), and it is also shown to inhibit the expression of LC3 II and ATGs, such as ATG5, ATG12 and ATG3 in hepatocellular carcinoma (47). In our study, we observed that knockdown of Cav1 abrogated IRGM expression, while IRGM overexpression reversed the downregulation of LC3 II, Beclin-1 and p62 caused by the knockdown of Cav1 expression. Furthermore, upregulation of IRGM could rescued the inhibition of cell death caused by downregulation of Cav1. These results demonstrate that downregulation of Cav1 alleviates the IR-resistant features in NSCLC cells through IRGM-regulated autophagy.

As a limitation, there is no other evidence indicating the relationship between Cav1 and IRGM in cell autophagy. Further in-depth studies are needed to explore the mechanism by which the Cav1-IRGM axis regulates autophagy in IR-resistant NSCLC cells and to verify this biological function in vivo. In addition, since p62 were downregulated after Cav1 knockdown, whether Cav1 could involve in selective autophagy in cancer cells deserves further study.

\section{Conclusions}

In summary, in this study, we identified a novel mechanism for acquired radioresistance in NSCLC cells. Elevated Cav1 expression was detected in IR-resistant NSCLC and functions as a promoter of radiation resistance that is dependent on IRGM-regulated autophagy. Our findings provide novel insights into the roles of Cav1 in promoting NSCLC resistance against IR and indicate that Cav1 could serve as a drug target for improving radiosensitivity. 


\section{Acknowledgments}

We all thank the members of the Center for Molecular Medicine for their critical comments.

Funding: This work was supported by grants from the Natural Science Foundation of Hunan Province (2020JJ5934, 2019JJ50932), the National Natural Science Foundation of China (No. 81703036, 81803035), China Postdoctoral Science Foundation (2020M672521, 2017M610510) and the Fundamental Research Funds for the Central Universities of Central South University (2019zzts800).

\section{Footnote}

Data Sharing Statement: Available at http://dx.doi. org/10.21037/atm-20-3293

Conflicts of Interest: All authors have completed the ICMJE uniform disclosure form (available at http://dx.doi. org/10.21037/atm-20-3293). Yuanliang Yan and Zhijie Xu serve as an unpaid section editor of Annals of Translational Medicine from Oct 2019 - Sep 2020. The other authors have no conflicts of interest to declare.

Ethical Statement: The authors are accountable for all aspects of the work in ensuring that questions related to the accuracy or integrity of any part of the work are appropriately investigated and resolved.

Open Access Statement: This is an Open Access article distributed in accordance with the Creative Commons Attribution-NonCommercial-NoDerivs 4.0 International License (CC BY-NC-ND 4.0), which permits the noncommercial replication and distribution of the article with the strict proviso that no changes or edits are made and the original work is properly cited (including links to both the formal publication through the relevant DOI and the license). See: https://creativecommons.org/licenses/by-nc-nd/4.0/.

\section{References}

1. Hirsch FR, Scagliotti GV, Mulshine JL, et al. Lung cancer: current therapies and new targeted treatments. Lancet 2017;389:299-311.

2. Zhang C, Leighl NB, Wu YL, et al. Emerging therapies for non-small cell lung cancer. J Hematol Oncol 2019;12:45.
3. Herbst RS, Morgensztern D, Boshoff C. The biology and management of non-small cell lung cancer. Nature 2018;553:446-54.

4. Oberndorfer F, Mullauer L. Molecular pathology of lung cancer: current status and perspectives. Curr Opin Oncol 2018;30:69-76.

5. Woodard GA, Jones KD, Jablons DM. Lung Cancer Staging and Prognosis. Cancer Treat Res 2016;170:47-75.

6. Yan Y, Zeng S, Wang X, et al. A Machine Learning Algorithm for Predicting Therapeutic Response to Anti-PD1. Technol Cancer Res Treat 2019;18:1533033819875766.

7. Yan Y, Chen X, Wei J, et al. Immunotherapy Combinations in Patients with Small Cell Lung Cancers. J Thorac Oncol 2019;14:e244-5.

8. Chen N, Wu L, Yuan H, et al. ROS/Autophagy/Nrf2 Pathway Mediated Low-Dose Radiation Induced RadioResistance in Human Lung Adenocarcinoma A549 Cell. Int J Biol Sci 2015;11:833-44.

9. Ren J, Liu T, Han Y, et al. GSK-3beta inhibits autophagy and enhances radiosensitivity in non-small cell lung cancer. Diagn Pathol 2018;13:33.

10. Karagounis IV, Kalamida D, Mitrakas A, et al. Repression of the autophagic response sensitises lung cancer cells to radiation and chemotherapy. Br J Cancer 2016;115:312-21.

11. Hurley JH, Young LN. Mechanisms of Autophagy Initiation. Annu Rev Biochem 2017;86:225-44.

12. Farias Quipildor GE, Mao K, Hu Z, et al. Central IGF1 protects against features of cognitive and sensorimotor decline with aging in male mice. Geroscience 2019;41:185-208.

13. Sarker MR, Franks SF. Efficacy of curcumin for ageassociated cognitive decline: a narrative review of preclinical and clinical studies. Geroscience 2018;40:73-95.

14. Souder DC, Anderson RM. An expanding GSK3 network: implications for aging research. Geroscience 2019;41:369-82.

15. Onorati AV, Dyczynski M, Ojha R, et al. Targeting autophagy in cancer. Cancer 2018;124:3307-18.

16. Yan Y, Chen X, Wang X, et al. The effects and the mechanisms of autophagy on the cancer-associated fibroblasts in cancer. J Exp Clin Cancer Res 2019;38:171.

17. Yan Y, Xu Z, Dai S, et al. Targeting autophagy to sensitive glioma to temozolomide treatment. J Exp Clin Cancer Res 2016;35:23.

18. Heine S, Kleih M, Gimenez N, et al. Cyclin D1-CDK4 activity drives sensitivity to bortezomib in mantle cell 
lymphoma by blocking autophagy-mediated proteolysis of NOXA. J Hematol Oncol 2018;11:112.

19. Chung C, Seo W, Silwal P, et al. Crosstalks between inflammasome and autophagy in cancer. J Hematol Oncol 2020;13:100.

20. Kao YC, Jheng JR, Pan HJ, et al. Elevated hydrostatic pressure enhances the motility and enlarges the size of the lung cancer cells through aquaporin upregulation mediated by caveolin-1 and ERK1/2 signaling. Oncogene 2017;36:863-74.

21. Liu L, Xu HX, Wang WQ, et al. Cavin-1 is essential for the tumor-promoting effect of caveolin-1 and enhances its prognostic potency in pancreatic cancer. Oncogene 2014;33:2728-36.

22. Campos A, Salomon C, Bustos R, et al. Caveolin-1containing extracellular vesicles transport adhesion proteins and promote malignancy in breast cancer cell lines. Nanomedicine (Lond) 2018;13:2597-609.

23. Zou M, Li Y, Xia S, et al. Knockdown of CAVEOLIN-1 Sensitizes Human Basal-Like Triple-Negative Breast Cancer Cells to Radiation. Cell Physiol Biochem 2017;44:778-91.

24. Chatterjee M, Ben-Josef E, Thomas DG, et al. Caveolin-1 is Associated with Tumor Progression and Confers a Multi-Modality Resistance Phenotype in Pancreatic Cancer. Sci Rep 2015;5:10867.

25. Yang X, Xiong H, Guan ZZ, et al. Higher expression of Caveolin-1 inhibits human small cell lung cancer (SCLC) apoptosis in vitro. Cancer Invest 2012;30:453-62 .

26. Dittmann K, Mayer C, Kehlbach R, et al. Radiationinduced caveolin-1 associated EGFR internalization is linked with nuclear EGFR transport and activation of DNA-PK. Mol Cancer 2008;7:69.

27. Yan Y, Xu Z, Qian L, et al. Identification of CAV1 and DCN as potential predictive biomarkers for lung adenocarcinoma. Am J Physiol Lung Cell Mol Physiol 2019;316:L630-43.

28. Duregon E, Senetta R, Pittaro A, et al. CAVEOLIN-1 expression in brain metastasis from lung cancer predicts worse outcome and radioresistance, irrespective of tumor histotype. Oncotarget 2015;6:29626-36.

29. Shi Y, Tan SH, Ng S, et al. Critical role of CAV1/ caveolin-1 in cell stress responses in human breast cancer cells via modulation of lysosomal function and autophagy. Autophagy 2015;11:769-84.

30. Liu WR, Jin L, Tian MX, et al. Caveolin-1 promotes tumor growth and metastasis via autophagy inhibition in hepatocellular carcinoma. Clin Res Hepatol Gastroenterol
2016;40:169-78.

31. Liu Y, Fu Y, Hu X, et al. Caveolin-1 knockdown increases the therapeutic sensitivity of lung cancer to cisplatininduced apoptosis by repressing Parkin-related mitophagy and activating the ROCK1 pathway. J Cell Physiol 2020;235:1197-208.

32. You S, Li R, Park D, et al. Disruption of STAT3 by niclosamide reverses radioresistance of human lung cancer. Mol Cancer Ther 2014;13:606-16.

33. Kiss T, Giles CB, Tarantini S, et al. Nicotinamide mononucleotide (NMN) supplementation promotes antiaging miRNA expression profile in the aorta of aged mice, predicting epigenetic rejuvenation and anti-atherogenic effects. Geroscience 2019;41:419-39.

34. Yan Y, Su W, Zeng S, et al. Effect and Mechanism of Tanshinone I on the Radiosensitivity of Lung Cancer Cells. Mol Pharm 2018;15:4843-53.

35. Uhlén M, Fagerberg L, Hallström BM, et al. Proteomics. Tissue-based map of the human proteome. Science 2015;347:1260419.

36. Győrffy B, Surowiak P, Budczies J, et al. Online survival analysis software to assess the prognostic value of biomarkers using transcriptomic data in non-small-cell lung cancer. PLoS One 2013;8:e82241.

37. Chauhan S, Mandell MA, Deretic V. IRGM governs the core autophagy machinery to conduct antimicrobial defense. Mol Cell 2015;58:507-21.

38. Dong Y, Digman MA, Brewer GJ. Age- and AD-related redox state of $\mathrm{NADH}$ in subcellular compartments by fluorescence lifetime imaging microscopy. Geroscience 2019;41:51-67.

39. Santín-Márquez R, Alarcón-Aguilar A, LópezDiazguerrero NE, et al. Sulforaphane - role in aging and neurodegeneration. Geroscience 2019;41:655-70.

40. Rožman P. The potential of non-myeloablative heterochronous autologous hematopoietic stem cell transplantation for extending a healthy life span. Geroscience 2018;40:221-42.

41. Kabeya Y, Mizushima N, Ueno T, et al. LC3, a mammalian homologue of yeast Apg8p, is localized in autophagosome membranes after processing. EMBO J 2000;19:5720-8.

42. Bekpen C, Xavier RJ, Eichler EE. Human IRGM gene "to be or not to be". Semin Immunopathol 2010;32:437-44.

43. Mehto S, Jena KK, Nath P, et al. The Crohn's Disease Risk Factor IRGM Limits NLRP3 Inflammasome Activation by Impeding Its Assembly and by Mediating Its Selective Autophagy. Mol Cell. 2019;73:429-45.e7.

44. Song Z, Guo C, Zhu L, et al. Elevated expression of 
immunity-related GTPase family $\mathrm{M}$ in gastric cancer.

Tumour Biol 2015;36:5591-6.

45. Xu Y, Liu R, Liao C, et al. High expression of immunityrelated GTPase family $M$ protein in glioma promotes cell proliferation and autophagy protein expression. Pathol Res Pract 2019;215:90-6.

46. Dong H, Tian L, Li R, et al. IFNg-induced Irgm1

Cite this article as: Chen X, Yan YL, Zeng SS, Gong ZC, $\mathrm{Xu}$ ZJ. Caveolin-1 promotes radioresistance via IRGMregulated autophagy in lung cancer. Ann Transl Med 2021;9(1):47. doi: 10.21037/atm-20-3293 promotes tumorigenesis of melanoma via dual regulation of apoptosis and Bif-1-dependent autophagy. Oncogene 2015;34:5363-71.

47. Wang LL, Jin XH, Cai MY, et al. AGBL2 promotes cancer cell growth through IRGM-regulated autophagy and enhanced Aurora A activity in hepatocellular carcinoma. Cancer Lett 2018;414:71-80. 